\title{
Junctions of mass-deformed nonlinear sigma models on the Grassmann manifold
}

\section{Sunyoung Shin}

Institute of Basic Science, Sungkyunkwan University, Suwon 16419, Republic of Korea

E-mail: sihnsy@skku.edu

ABSTRACT: We study vacua and walls of the mass-deformed nonlinear sigma models on the Grassmann manifold $G_{N_{F}, N_{C}}=\frac{\mathrm{SU}\left(N_{F}\right)}{\mathrm{SU}\left(N_{C}\right) \times \mathrm{SU}\left(N_{F}-N_{C}\right) \times \mathrm{U}(1)}$ and discuss three-pronged junctions for $N_{C}=1,2,3$ in four dimensions.

KeYwords: Solitons Monopoles and Instantons, Extended Supersymmetry, Sigma Models ARXIV EPRINT: 1904.12823 


\section{Contents}

1 Introduction 1

2 Model 3

$\begin{array}{llc}3 & \text { Vacua and walls } & 6\end{array}$

4 Three-pronged junctions $\quad 10$

5 Summary and discussion $\quad 14$

A Diagram for vacua and elementary walls of the mass-deformed nonlinear sigma models on $G_{8,4} \quad 14$

\section{Introduction}

Topological defects play important roles in model building. Our world can be realized on extended topological defects such as walls or junctions in brane world scenarios [1-4]. Wall networks are applied to study dark matter and dark energy [5-10].

Walls interpolating isolated supersymmetric vacua preserve $1 / 2$ supersymmetry. Walls in Abelian gauge theories are studied in [11-18]. The moduli matrix formalism is proposed to analyse walls of $\mathcal{N}=2$ non-Abelian gauge theories $[19,20]$. In the strong coupling limit, the model with $N_{F}>N_{C}$ becomes the mass-deformed hyper-Kähler nonlinear sigma model on $T^{*} G_{N_{F}, N_{C}}$. In this limit, the wall solutions become exact. It is shown that with the Fayet-Iliopoulos parameters $\left(b, b^{*}, c\right)=(0,0, c>0)$, we can find a bundle structure so that vacua and walls are on the Kähler manifold, which can be realized as the $\mathcal{N}=1$ non-Abelian gauge theory. The moduli matrix formalism is thoroughly examined in the mass-deformed nonlinear sigma models on the Grassmann manifold, which produce exact Bogomol'nyi-Prasad-Sommerfield (BPS) solutions [19, 20].

We can consider models with superpotentials as vacua and walls of the mass-deformed nonlinear sigma models can be analytically analysed on the Grassmann manifold. The simplest models that have exact BPS solutions are the mass-deformed nonlinear sigma models on the quadrics of the Grassmann manifold $\mathrm{SO}(2 N) / \mathrm{U}(N)$ and $\operatorname{Sp}(N) / \mathrm{U}(N)$. The nonlinear sigma models on $\mathrm{SO}(2 N) / \mathrm{U}(N)$ and $\mathrm{Sp}(N) / \mathrm{U}(N)$ are constructed as gauge theories by (anti-)holomorphic embeddings to the Grassmann manifold [21]. By integrating out the Lagrange multipliers of the superpotential terms, we obtain the F-term constraints. By introducing mass-terms to the models, the most part of the continuous vacuum is lifted and a finite number of discrete vacua remain on the surfaces. We can find exact BPS solutions that interpolate the discrete vacua. 
In the moduli matrix formalism, walls are algebraically constructed from elementary walls. The elementary walls can be identified with simple roots of the global symmetry [22]. In $[23,24]$, a pictorial representation is proposed to study vacua and walls of mass-deformed Kähler nonlinear sigma models on $\mathrm{SO}(2 N) / \mathrm{U}(N)$ and $\mathrm{Sp}(N) / \mathrm{U}(N)$, which are quadrics of the Grassmann manifold $G_{2 N, N}$. It is observed that we can produce the whole structure of vacua and elementary walls from the vacuum structures that are connected to the maximum number of elementary walls for generic $N$. There is a recurrence of a two dimensional diagram for each $N$ mod 4 in the vacuum structures on $\mathrm{SO}(2 N) / \mathrm{U}(N)$ and $\operatorname{Sp}(N) / \mathrm{U}(N)$. The vacuum structures are proved by induction.

The observation made in [23, 24] should be useful not only for constructing BPS walls, but also for analysing supersymmetric models, which have 1/1 vacua, 1/2 BPS objects and $1 / 4$ BPS objects since the moduli matrix formalism produces exact solutions in the mass-deformed nonlinear sigma models on the compact Hermitian symmetric spaces of the quotient with respect to the gauge group.

There are two standard off-shell $\mathcal{N}=2$ superspace formalisms. One is the harmonic superspace formalism and the other is the projective superspace formalism. In the harmonic superspace formalism, the nonlinear sigma models on the complex projective spaces and on the Grassmann manifold are constructed as the quotient with respect to the gauge group [25-27], but to the best of the author's knowledge, it is not known whether (anti)holomorphic embeddings can be defined. In the projective superspace formalism, the holomorphic embeddings for the nonlinear sigma models on the compact Hermitian symmetric spaces are well-defined but the models are constructed in the inhomogeneous coordinates of the target space [28]. Therefore provided that we are willing to construct models of the quotient with respect to the gauge group, it is useful to study vacua and BPS objects of the $\mathcal{N}=2$ mass-deformed nonlinear sigma models on the quadrics of the Grassmann manifold in the $\mathcal{N}=1$ superspace formalism to get better understanding of the $\mathcal{N}=2$ supersymmetric model building.

Intersecting walls form junctions, which preserve $1 / 4$ supersymmetry [29-38]. The energy density of a wall junction is bounded from below by central charge densities $\mathcal{Z}_{\alpha}$ $(\alpha=1,2)$ and $\mathcal{Y} . \mathcal{Z}_{\alpha}$ are components of the tension vector of the wall, which pulls the junction along the wall direction outwards and $\mathcal{Y}$ is the charge density for the junction. An Abelian junction has a negative $Y$-charge while a non-Abelian junction has a positive $Y$-charge. In the strong coupling limit, the charge density $\mathcal{Y}$ vanishes.

A three-pronged junction is formed by a set of three vacua that are interpolated by walls. Three-pronged junction solutions are discussed in the moduli matrix formalism [3941]. The moduli matrix formalism can be easily applied to Abelian junctions, but it becomes complicated with non-Abelian junctions. In [40], three-pronged junctions of massdeformed nonlinear sigma models on the Grassmann manifold $G_{N_{F}, N_{C}}$ are analyzed by the Plücker embedding. The models are embedded into the complex projective space $\mathbf{C} P^{N_{F}} C_{N_{C}}-1$, which produce Abelian gauge theories. The Plücker embedding is useful for the Grassmann manifold, but the drawback of it is that the method is not directly applicable to the quadrics of the Grassmann manifold.

An alternative method is proposed to construct three-pronged junctions of the massdeformed nonlinear sigma models on the Grassmann manifold [42]. The pictorial repre- 
sentation, which is introduced by [23, 24], is applied to vacua and walls of mass-deformed nonlinear sigma models on the Grassmann manifold. The vacua and the walls of the massdeformed nonlinear sigma models on $G_{N_{F}, N_{C}}$ with $\left(N_{F}, N_{C}\right)=(4,2),(5,2),(5,3),(6,2)$, $(6,3),(6,4)$ are explicitly shown in the representation. In the representation, the duality of the Grassmann manifold $N_{C} \leftrightarrow N_{F}-N_{C}$ is realized as a $\pi$-rotation of the diagram. The diagram of $G_{N_{F}+1, N_{C}}$ repeats the diagram of $G_{N_{F}, N_{C}}$. It is also shown in [42] that by reformulating the diagrams in the pictorial representations, we can produce polyhedra, which are similar to the polyhedra that are introduced to study BPS objects of the massdeformed nonlinear sigma models on the complex projective space [39]. Vertices, edges and triangular faces correspond to vacua, walls and three-pronged junctions. Positions of three-pronged junctions are computed by making use of the polyhedra instead of using the Plüker embedding [42].

In this paper, we discuss vacua, walls and three-pronged junctions of mass-deformed nonlinear sigma models on the Grassmann manifold with flavour number $N_{F}$ and colour number $N_{C}=1,2,3$. Since diagrams in the pictorial representation consist of the simple roots of $\mathrm{SU}\left(N_{F}\right)$, diagrams for $N_{C} \geq 4$ are trivial extensions.

This paper is organized as follows. In section 2, we review the model [43-45] and the moduli matrix formalism [19, 20]. In section 3, we review the work [42] and discuss vacua and walls of the mass-deformed nonlinear sigma models on $G_{N_{F}, N_{C}}$ with $N_{C}=1,2,3$. In section 4, we apply the method, which is introduced by [42], to three-pronged junctions of the mass-deformed nonlinear sigma model on $G_{N_{F}, 3}$. In section 5, we summarize our results. In $\mathrm{A}$, we present the diagram for $G_{8,4}$ to show that the method discussed in section 3 is generically applicable.

\section{Model}

We study junctions of the $\mathcal{N}=2$ mass-deformed nonlinear sigma model on the Grassmann manifold $G_{N_{F}, N_{C}}=\frac{\mathrm{SU}\left(N_{F}\right)}{\mathrm{SU}\left(N_{C}\right) \times \mathrm{SU}\left(N_{F}-N_{C}\right) \times \mathrm{U}(1)}$ in four dimensions. The Lagrangian of the mass-deformed nonlinear sigma model [43-45] is

$$
\begin{aligned}
\mathcal{L}= & \int d^{4} \theta \operatorname{Tr}\left[\Phi \Phi^{\dagger} e^{V}+\Psi^{\dagger} \Psi e^{-V}-c V\right] \\
& +\int d^{2} \theta \operatorname{Tr}\left[\Lambda\left(\Phi \Psi-b I_{M}\right)+\Phi \mathcal{M} \Psi+(\text { conjugate transpose })\right], \\
& \left(c \in \mathbf{R}_{\geq 0}, b \in \mathbf{C}\right),
\end{aligned}
$$

where $c$ is the electric Fayet-Iliopoulos (FI) parameter and $b, b^{*}$ are the magnetic FI parameters. Chiral fields $\Phi_{a}^{i}(x, \theta, \bar{\theta}), \Psi_{i}{ }^{a}(x, \theta, \bar{\theta}), \Lambda_{a}^{b}$ and vector field $V_{a}{ }^{b}(x, \theta, \bar{\theta})$ are matrix valued and defined as follows:

$$
\begin{aligned}
& \Phi_{a}^{i}(y)=\phi_{a}{ }^{i}(y)+\sqrt{2} \theta \psi_{a}{ }^{i}(y)+\theta \theta F_{a}{ }^{i}(y), \quad\left(y^{\mu}=x^{\mu}+i \theta \sigma^{\mu} \bar{\theta}\right) \\
& \Psi_{i}{ }^{a}(y)=\varphi_{i}{ }^{a}(y)+\sqrt{2} \theta \chi_{i}{ }^{a}(y)+\theta \theta G_{i}{ }^{a}(y) \\
& V_{a}{ }^{b}(x)=2 \theta \sigma^{\mu} \bar{\theta} A_{\mu a}{ }^{b}(x)+i \theta \theta \bar{\theta} \bar{\lambda}_{a}^{b}(x)-i \bar{\theta} \bar{\theta} \theta \lambda_{a}^{b}(x)+\theta \theta \bar{\theta} \bar{\theta} D_{a}^{b}(x) \\
& \Lambda_{a}{ }^{b}(y)=-\mathcal{S}_{a}{ }^{b}(y)+\theta \eta_{a}{ }^{b}(y)+\theta \theta K_{a}{ }^{b}(y), \\
&\left(a=1, \cdots, N_{C} ; i=1, \cdots, N_{F} ; \mu=0, \cdots, 3\right) .
\end{aligned}
$$

We diagonalise $V$ and $\Lambda$ for later use. 
The $\mathrm{SU}(N)$ Cartan generators $\underline{H}=\left(H_{1}, \cdots, H_{N}\right)[46]$ are defined by

$$
H_{n}=e_{n, n}-\frac{1}{N} I_{N \times N}, \quad(n=1, \cdots, N),
$$

where $e_{p, q}$ is an $N \times N$ matrix of which the $(p, q)$ component is one. The complex mass matrix $\mathcal{M}$ can be formulated as a linear combination of (2.3) with complex parameters. The mass matrix $\mathcal{M}$ is a traceless diagonal matrix.

The Lagrangian (2.1) with the component fields (2.2) can be computed. The equations of the auxiliary fields are solved by

$$
\begin{array}{ll}
F=-\varphi^{\dagger} \mathcal{M}^{\dagger}+\mathcal{S}^{\dagger} \varphi^{\dagger}, & \text { (conjugate transpose) } \\
G=-\mathcal{M}^{\dagger} \phi^{\dagger}+\phi^{\dagger} \mathcal{S}^{\dagger}, & \text { (conjugate transpose) }
\end{array}
$$

Then the bosonic part of the Lagrangian is

$$
\begin{aligned}
\mathcal{L}=\operatorname{Tr}[ & D_{\mu} \phi\left(D^{\mu} \phi\right)^{\dagger}+\left(D_{\mu} \varphi\right)^{\dagger} D^{\mu} \varphi-|\phi \mathcal{M}-\mathcal{S} \phi|^{2}-|\mathcal{M} \varphi-\varphi \mathcal{S}|^{2} \\
& \left.+\left(\phi \phi^{\dagger}-\varphi^{\dagger} \varphi-c I_{N_{C}}\right) D+K\left(\phi \varphi-b I_{N_{C}}\right)+\left(\varphi^{\dagger} \phi^{\dagger}-b^{*} I_{N_{C}}\right) K^{\dagger}\right] .
\end{aligned}
$$

The covariant derivatives are defined by $D_{\mu} \phi=\partial_{\mu} \phi-i A_{\mu} \phi$ and $D_{\mu} \varphi=\partial_{\mu} \varphi+i \varphi A_{\mu}$. The Lagrangian has constraints

$$
\begin{aligned}
\phi \phi^{\dagger}-\varphi^{\dagger} \varphi-c I_{N_{C}} & =0, \\
\phi \varphi-b I_{N_{C}} & =0, \quad \text { (conjugate transpose). }
\end{aligned}
$$

There are two cases for the constraint (2.7), $b=0$ and $b \neq 0$, which are related by the $\mathrm{SU}(2)_{R}$ symmetry. We consider $b=0$ case in this paper. In this case, field $\phi$ parameterizes the base space of the Grassmann manifold whereas field $\varphi$ parameterizes the cotangent space. Field $\varphi$ does not contribute to the vacuum configuration and the BPS solutions of the mass-deformed nonlinear sigma models on the Grassmann manifold [19, 20, 39]. Then the relevant bosonic part of the Lagrangian (2.5) is

$$
\mathcal{L}=\operatorname{Tr}\left[D_{\mu} \phi\left(D^{\mu} \phi\right)^{\dagger}-|\phi \mathcal{M}-\mathcal{S} \phi|^{2}+\phi \phi^{\dagger} D-c D\right] .
$$

The Lagrangian has a constraint

$$
\phi \phi^{\dagger}-c I_{N_{C}}=0
$$

By substituting $\mathcal{M}$ and $\mathcal{S}$ in the Lagrangian (2.8) with real valued matrices $\widetilde{M}_{\alpha}$ and $\widetilde{\Sigma}_{\alpha}$, $(\alpha=1,2)$ as

$$
\mathcal{M}=\widetilde{M}_{1}+i \widetilde{M}_{2}, \quad \mathcal{S}=\widetilde{\Sigma}_{1}+i \widetilde{\Sigma}_{2},
$$

we get the vacuum condition

$$
\phi \widetilde{M}_{a}-\widetilde{\Sigma}_{a} \phi=0, \quad(a=1,2) .
$$


The mass matrices and the real scalar fields can be parameterized as

$$
\begin{aligned}
& \widetilde{M}_{1}=\operatorname{diag}\left(l_{1}, l_{2}, \cdots, l_{N_{F}}\right), \\
& \widetilde{M}_{2}=\operatorname{diag}\left(n_{1}, n_{2}, \cdots, n_{N_{F}}\right), \\
& \widetilde{\Sigma}_{1}=\operatorname{diag}\left(\sigma_{1}, \sigma_{2}, \cdots, \sigma_{N_{C}}\right), \\
& \widetilde{\Sigma}_{2}=\operatorname{diag}\left(\tau_{1}, \tau_{2}, \cdots, \tau_{N_{C}}\right) .
\end{aligned}
$$

Then the vacuum solutions are labelled by

$$
\left(\sigma_{1}+i \tau_{1}, \sigma_{2}+i \tau_{2}, \cdots, \sigma_{N_{C}}+i \tau_{N_{C}}\right)=\left(l_{i}+i n_{i}, l_{j}+i n_{j}, \cdots, l_{k}+i n_{k}\right)
$$

where $i, j, k=1, \cdots, N_{F}$. Therefore there are ${ }_{N_{F}} C_{N_{C}}$ vacuum solutions as it is observed in [45]. The vacuum solutions should be constrained by (2.9).

We are interested in static configurations, which are independent of the $x^{3}$-coordinate. So we fix $\partial_{0}=\partial_{3}=0$. We also assume that there is the Poincare invariance on the worldvolume so we fix $A_{0}=A_{3}=0$. Then the energy density is

$$
\mathcal{E}=\operatorname{Tr}\left[\sum_{\alpha=1,2}\left|D_{\alpha} \phi \mp\left(\phi \widetilde{M}_{\alpha}-\widetilde{\Sigma}_{\alpha} \phi\right)\right|^{2}\right] \pm \mathcal{T} \geq \pm \mathcal{T},
$$

where the tension density is

$$
\mathcal{T}=\operatorname{Tr}\left[\sum_{\alpha=1,2} \partial_{\alpha}\left(\phi \widetilde{M}_{\alpha} \phi^{\dagger}\right)\right] .
$$

We use the index $\alpha=1,2$ for codimensions and adjoint scalars as it is done in [39]. The energy density (2.14) and the tension density (2.15) are constrained by (2.9).

The (anti-)BPS equation is

$$
D_{\alpha} \phi \mp\left(\phi \widetilde{M}_{\alpha}-\widetilde{\Sigma}_{\alpha} \phi\right)=0, \quad(\alpha=1,2) .
$$

We choose the upper sign for the BPS equation and the lower sign for the anti-BPS equation. The BPS solution $[19,20,39,40]$ is

$$
\phi=S^{-1} H_{0} e^{\widetilde{M}_{1} x^{1}+\widetilde{M}_{2} x^{2}},
$$

with a relation

$$
S^{-1} \partial_{\alpha} S:=\widetilde{\Sigma}_{\alpha}-i A_{\alpha}, \quad(\alpha=1,2) .
$$

The constraint (2.9) becomes

$$
S S^{\dagger}=\frac{1}{c} H_{0} e^{2 \widetilde{M}_{1} x^{1}+2 \widetilde{M}_{2} x^{2}} H_{0}^{\dagger} .
$$

The BPS solution (2.17), $\Sigma_{\alpha}$ and $A_{\alpha}$ in (2.18) are invariant under the following transformation:

$$
H_{0}^{\prime}=V H_{0}, \quad S^{\prime}=V S, \quad V \in \mathrm{GL}\left(N_{C}, \mathbf{C}\right)
$$


This equivalent class of $\left(S, H_{0}\right)$ is called worldvolume symmetry in the moduli matrix formalism $[19,20]$. Therefore the moduli space, which is parameterized by $H_{0}$, is the Grassmann manifold.

1/1 BPS supersymmetric vacua and 1/2 BPS walls can be constructed by a model with real masses and real fields $[19,20]$. The $1 / 4$ BPS junctions are constructed by a model with complex masses and complex fields [39, 40].

\section{$3 \quad$ Vacua and walls}

We study vacua and walls of the mass-deformed nonlinear sigma models on the Grassmann manifold by using the moduli matrix formalism [19, 20] and the pictorial representation [23, $24,42]$. We can simplify the Lagrangian (2.8) by introducing a real mass matrix and a real scalar matrix field. The vacuum condition is

$$
\phi M-\Sigma \phi=0 .
$$

$M$ and $\Sigma$ are related to $\mathcal{M}$ and $\mathcal{S}$ in (2.8) [39] by

$$
\begin{aligned}
\mathcal{M} & =e^{i \theta}\left(M-\Delta M I_{N_{F}}\right), \\
\mathcal{S} & =e^{i \theta}\left(\Sigma-\Delta M I_{N_{C}}\right) .
\end{aligned}
$$

The matrices can be parameterized as

$$
\begin{aligned}
M & =\operatorname{diag}\left(m_{1}, m_{2}, \cdots, m_{N_{F}}\right), \\
\Sigma & =\operatorname{diag}\left(\sigma_{1}, \sigma_{2}, \cdots, \sigma_{N_{C}}\right) .
\end{aligned}
$$

We can set $m_{1}>m_{2}>\cdots>m_{N}$ without loss of generality since we are interested in generic mass parameters. Then the vacuum solutions are labelled by

$$
\left(\sigma_{1}, \sigma_{2}, \cdots, \sigma_{N_{C}}\right)=\left(m_{i}, m_{j}, \cdots, m_{k}\right),
$$

where $i, j, k=1, \cdots, N_{F}$. The vacuum solutions are the same as the ones labelled by (2.13).

We study walls. We can assume that fields are static and all the fields depend on $x_{1} \equiv x$ coordinate. We can also assume that there is the Poincare invariance on the worldvolume of walls so we set $A_{0}=A_{2}=A_{3}=0$. We can learn from (2.17) that the BPS solution $[19,20]$ is

$$
\phi=S^{-1} H_{0} e^{M x}
$$

Walls are constructed from elementary walls in the moduli matrix formalism. Let $\langle A\rangle$ denote a vacuum and $\langle A \leftarrow B\rangle$ denote a wall that connects vacuum $\langle A\rangle$ and vacuum $\langle B\rangle$. The moduli matrix of elementary wall $\langle A \leftarrow B\rangle$ is

$$
\begin{aligned}
H_{0\langle A \leftarrow B\rangle} & =H_{0\langle A\rangle} e^{E_{i}(r)}, \\
E_{i}(r) & \equiv e^{r} E_{i}, \quad(i=1, \cdots, N),
\end{aligned}
$$


where $E_{i}$ is an elementary wall operator and $r$ is a complex parameter with $-\infty<$ $\operatorname{Re}(r)<+\infty$. The elementary wall operator $E_{i}$ is a simple root generator of $\operatorname{SU}\left(N_{F}\right)$, which satisfies

$$
c\left[M, E_{i}\right]=c\left(\underline{m} \cdot \underline{a}_{i}\right) E_{i}=T_{\langle A \leftarrow B\rangle} E_{i},
$$

where $c$ is the electric FI parameter of the Lagrangian $(2.1), \underline{m}=\left(m_{1}, \cdots, m_{N_{F}}\right)$, and $T_{\langle A \leftarrow B\rangle}$ is the tension of the elementary wall. Elementary walls can be identified with the simple roots of $\mathrm{SU}\left(N_{F}\right)[22]$.

The simple root generators and the simple roots of $\mathrm{SU}(N)[46]$ are

$$
\begin{aligned}
E_{i} & =e_{i, i+1}, \\
\underline{\alpha}_{i} & =\hat{e}_{i}-\hat{e}_{i+1}, \quad(i=1, \cdots, N-1) .
\end{aligned}
$$

The set of vectors $\left\{\hat{e}_{i}\right\}$ is the orthogonal unit vectors $\hat{e}_{i} \cdot \hat{e}_{j}=\delta_{i j}$.

Elementary walls can be compressed to single walls. A compressed wall of level $n$ which connects $\langle A\rangle$ and $\left\langle A^{\prime}\right\rangle$ is

$$
\begin{aligned}
H_{0\left\langle A \leftarrow A^{\prime}\right\rangle}=H_{0\langle A\rangle} e^{\left[E_{i_{1}},\left[E_{i_{2}},\left[E_{i_{3}},\left[\cdots,\left[E_{i_{n}}, E_{i_{n+1}}\right] \cdots\right]\right]\right](r)\right.}, \\
\left(i_{m}=1, \cdots, N ; m=1, \cdots, n+1\right) .
\end{aligned}
$$

A multiwall is constructed by multiplying a single wall operator to another wall moduli matrix. A multiwall interpolating $\langle A\rangle,\left\langle A^{\prime}\right\rangle, \cdots$, and $\langle B\rangle$ is

$$
\begin{aligned}
H_{0\left\langle A \leftarrow A^{\prime} \leftarrow \cdots \leftarrow B\right\rangle}=H_{0\langle A\rangle} e^{E_{i_{1}}\left(r_{1}\right)} e^{E_{i_{2}}\left(r_{2}\right)} \cdots e^{E_{i_{n}}\left(r_{n}\right)}, \\
\left(i_{m}=1, \cdots, N ; m=1, \cdots, n\right),
\end{aligned}
$$

where parameters $r_{i},(i=1,2, \cdots)$ are complex parameters with $-\infty<\operatorname{Re}\left(r_{i}\right)<+\infty$. Penetrable walls pass through each other since the wall operators commute:

$$
\left[E_{i_{m}}, E_{i_{n}}\right]=0 .
$$

Let vector $\underline{g}_{\left\langle A \leftarrow A^{\prime}\right\rangle}$ denote the wall that interpolates vacuum $\langle A\rangle$ and vacuum $\left\langle A^{\prime}\right\rangle$. Then the elementary wall (3.6) with the relation (3.7) can be identified with

$$
\underline{g}_{\langle A \leftarrow B\rangle} \equiv c \underline{\alpha}_{i} .
$$

The tension of the wall can be read from (3.7) as

$$
T_{\left\langle A \leftarrow A^{\prime}\right\rangle}=\underline{m} \cdot \underline{g}_{\left\langle A \leftarrow A^{\prime}\right\rangle} \cdot
$$

The compressed wall in (3.9) is identified with

$$
\underline{g}_{\left\langle A \leftarrow A^{\prime}\right\rangle} \equiv c \underline{\alpha}_{i_{1}}+c \underline{\alpha}_{i_{2}}+c \underline{\alpha}_{i_{3}}+\cdots+c \underline{\alpha}_{i_{n}}+c \underline{\alpha}_{i_{n+1}} .
$$

The root vectors of the two penetrable walls of (3.11) are orthogonal

$$
\underline{g}_{i_{m}} \cdot \underline{g}_{i_{n}}=0
$$




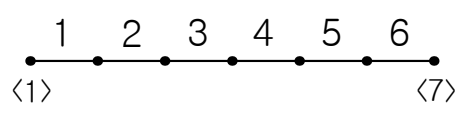

(a)

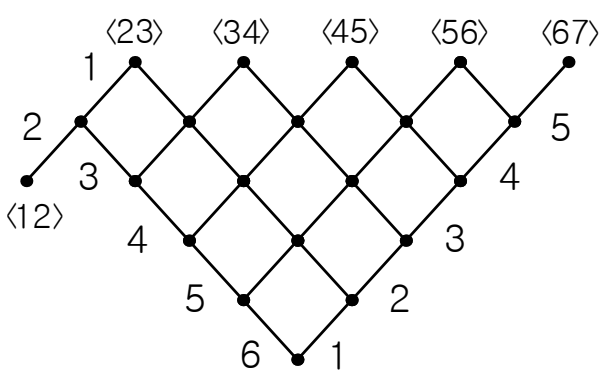

(b)

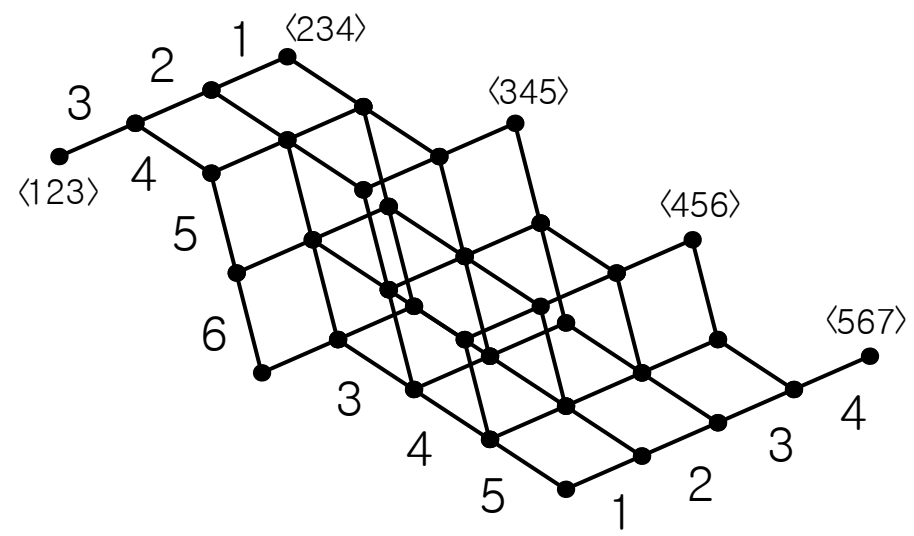

(c)

Figure 1. Vacua and elementary walls in $G_{7, N}$. The numbers indicate the subscript $i$ 's of simple $\operatorname{roots} \underline{\alpha}_{i}$. (a) $G_{7,1}$ (b) $G_{7,2}$ (c) $G_{7,3}$.

The pictorial representation of $[23,24]$ is applied to vacua and walls of mass-deformed nonlinear sigma models on the Grassmann manifold in [42]. In the representation, vacua and elementary walls are described by vertices and segments. It is observed that the duality $N_{C} \leftrightarrow N_{F}-N_{C}$ corresponds to a $\pi$-rotation in the pictorial representation and the diagram of $G_{N_{F}+1, N_{C}}$ repeats the diagram of $G_{N_{F}, N_{C}}$. The diagrams of the vacua and the elementary walls of the mass-deformed nonlinear sigma models on $G_{N_{F}, N_{C}}$, with $\left(N_{F}, N_{C}\right)=(4,2),(5,2),(5,3),(6,2),(6,3),(6,4)$ are shown in [42]. The diagrams of the vacua and the elementary walls of the mass-deformed nonlinear sigma models on $G_{7, N_{C}}$ with $N_{C}=1,2,3$ are presented in figure 1 .

The mass-deformed nonlinear sigma models on $G_{N_{F}, N_{C}}$ have ${ }_{N_{F}} C_{N_{C}}$ vacua. All the walls of the model are constructed from elementary walls, which can be described by the simple roots of $\mathrm{SU}\left(N_{F}\right), \underline{\alpha}_{i},\left(i=1, \cdots, N_{F}-1\right)$ of (3.8). As we can see in [42] and figure 1, the diagrams for $G_{N_{F}, N_{C}}$ repeats the diagrams for $G_{n, N_{C}},\left(N_{C}<n \leq N_{F}-1\right)$. It shows that vacua and elementary walls of the mass-deformed nonlinear sigma models on $G_{N_{F}+1, N_{C}}$ can be derived from the configuration of vacua and elementary walls of $G_{N_{F}, N_{C}}$, by adding ${ }_{N_{F}} C_{N_{C}-1}$ number of vacua and elementary walls. We can focus on the case where $N_{C} \leq\left[\frac{N_{F}}{2}\right]$ since the case with $N_{C}>\left[\frac{N_{F}}{2}\right]$ can be determined by the duality condition $G_{N_{F}, N_{C}} \simeq G_{N_{F}, N_{F}-N_{C}}$, which is related by a $\pi$-rotation in the pictorial representation. 


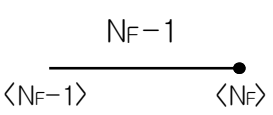

(a)

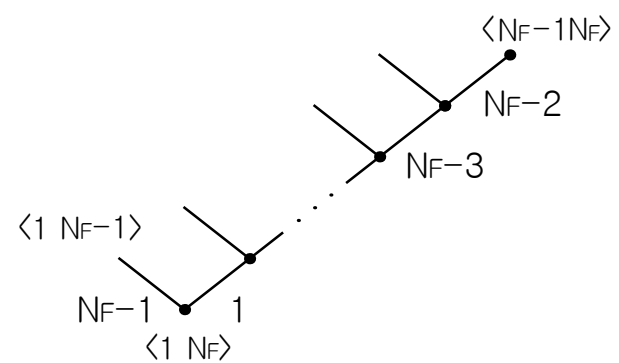

(b)

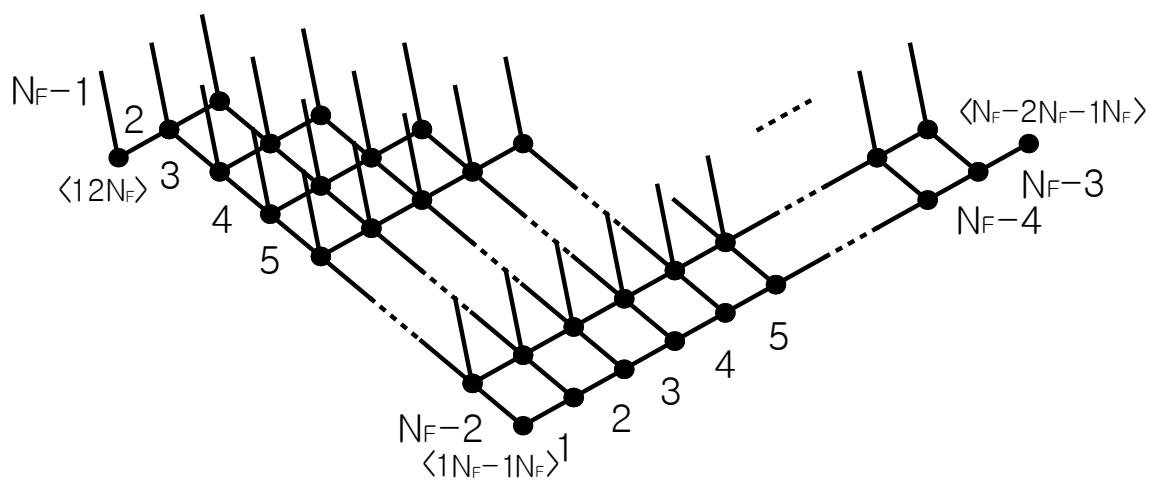

(c)

Figure 2. (a) $N_{C}=1 ;$ (b) $N_{C}=2 ;\left(\right.$ c) $N_{C}=3$.

We present diagrams for $N_{C}=1,2,3$ in figure 2 . The structure of vacua and elementary walls of the mass-deformed nonlinear sigma models on $G_{N_{F}, 1}$ is constructed by adding one vacuum $\left\langle N_{F}\right\rangle$ and one elementary wall $\underline{\alpha}_{N_{F}-1}$ to the structure of $G_{N_{F}-1,1}$. The diagram of the additional structure is depicted in figure 2(a). The structure of vacua and elementary walls of the mass-deformed nonlinear sigma models on $G_{N_{F}, 2}$ is constructed by adding the diagram in figure 2(b), which consists of $\left(N_{F}-1\right)$ vacua, $\left(N_{F}-2\right)$ number of $\underline{\alpha}_{N_{F}-1}$ and one for each $\underline{\alpha}_{i}, i=1, \cdots, N_{F}-2$, to the diagram for $G_{N_{F}-1,2}$. In the similar manner, the diagram of vacua and elementary walls of mass-deformed nonlinear sigma models on $G_{N_{F}, 3}$ is obtained by adding the diagram in figure 2(c) to the diagram for $G_{N_{F}-1,3}$. The diagram adds $\left(N_{F}-3\right)$ number of each $\underline{\alpha}_{i}\left(i=1, \cdots, N_{F}-2\right)$ and $\frac{\left(N_{F}-3\right)\left(N_{F}-2\right)}{2}$ number of $\underline{\alpha}_{N_{F}-1}$. The configuration of vacua and walls of the mass-deformed nonlinear sigma models on $G_{N_{F}, N_{C}}$ can be systematically derived from the diagrams of the pictorial representation.

We have discussed generic properties of vacua and elementary walls of the nonlinear sigma models on the Grassmann manifold with flavour number $N_{F}$ for colour number $N_{C}=1,2,3$ as examples. Non-orthogonal elementary walls are aligned next to each other. Orthogonal elementary walls form parallelograms. Since the global symmetry is $\mathrm{SU}\left(N_{F}\right)$, diagrams for $N_{C} \geq 4$ cases are trivial extensions. For a finite number of discrete vacua, we can always identify the simple roots of $\mathrm{SU}\left(N_{F}\right)$ that interpolate the vacua. We present the diagram for vacua and elementary walls of the mass-deformed nonlinear sigma model on $G_{8,4}$ as an example in A. 


\section{Three-pronged junctions}

A three-pronged junction is formed by three vacua interpolated by three non-penetrable walls. A wall that interpolates vacuum $\langle A\rangle$ and vacuum $\langle B\rangle$ has a tension $\underline{T}^{A B}$ pulling the junction along the wall direction outwards [39]

$$
\begin{aligned}
\underline{T}^{\langle A B\rangle} & =\left(-Z_{2}^{A B}, Z_{1}^{A B}\right), \\
\left(Z_{1}^{A B}, Z_{2}^{A B}\right) & \equiv c\left(l_{A}-l_{B}, n_{A}-n_{B}\right),
\end{aligned}
$$

which is computed by the line integral of the tension density defined by (2.15).

We study three-pronged junctions of the mass-deformed nonlinear sigma models on the Grassmann manifold $G_{N_{F}, 3}$. We reformulate the diagram in figure 2(c) by connecting adjacent vacua to produce a pyramid of which the vertices, the edges, the triangular faces and the parallelogram shaped base correspond to vacua, walls, three-pronged junctions and two pairs of penetrable walls [42]. ${ }^{1}$ The pyramid is depicted in figure 3. Let us use semicolons in vacuum labels $\langle i ; j ; \cdots ; k\rangle$ to separate the flavour numbers.

There are two types of junctions [40]. Abelian junctions divide a set of three vacua with labels that differ by one components: $\langle\cdots, A\rangle,\langle\cdots, B\rangle,\langle\cdots, C\rangle$. Non-Abelian junctions divide a set of three vacua with labels that differ by two components: $\langle\cdots, A B\rangle$, $\langle\cdots, B C\rangle,\langle\cdots, A C\rangle$. Abelian junctions exist in Abelian gauge theories and non-Abelian gauge theories whereas non-Abelian junctions exist only in non-Abelian gauge theories.

There are two Abelian junctions and two non-Abelian junctions in figure 3:

- Abelian junctions:

$$
\begin{aligned}
& \left\{\left\langle N_{F}-4 ; N_{F}-2 ; N_{F}\right\rangle,\left\langle N_{F}-3 ; N_{F}-2 ; N_{F}\right\rangle,\left\langle N_{F}-2 ; N_{F}-1 ; N_{F}\right\rangle\right\}, \\
& \left\{\left\langle N_{F}-4 ; N_{F}-1 ; N_{F}\right\rangle,\left\langle N_{F}-3 ; N_{F}-1 ; N_{F}\right\rangle,\left\langle N_{F}-2 ; N_{F}-1 ; N_{F}\right\rangle\right\} .
\end{aligned}
$$

- Non-abelian junctions:

$$
\begin{aligned}
& \left\{\left\langle N_{F}-4 ; N_{F}-2 ; N_{F}\right\rangle,\left\langle N_{F}-4 ; N_{F}-1 ; N_{F}\right\rangle,\left\langle N_{F}-2 ; N_{F}-1 ; N_{F}\right\rangle\right\}, \\
& \left\{\left\langle N_{F}-3 ; N_{F}-1 ; N_{F}\right\rangle,\left\langle N_{F}-3 ; N_{F}-2 ; N_{F}\right\rangle,\left\langle N_{F}-2 ; N_{F}-1 ; N_{F}\right\rangle\right\} .
\end{aligned}
$$

We study Abelian junction $\left\{\left\langle N_{F}-4 ; N_{F}-2 ; N_{F}\right\rangle,\left\langle N_{F}-3 ; N_{F}-2 ; N_{F}\right\rangle,\left\langle N_{F}-2 ; N_{F}-\right.\right.$ $\left.\left.1 ; N_{F}\right\rangle\right\}$. The moduli matrix for the junction is

$$
\begin{aligned}
H_{0} & =\left(\begin{array}{cccccc}
\cdots & h_{1 N_{F}-4} & h_{1 N_{F}-3} & 0 & h_{1 N_{F}-1} & 0 \\
\cdots & 0 & 0 & h_{2 N_{F}-2} & 0 & 0 \\
\cdots & 0 & 0 & 0 & 0 & h_{3 N_{F}}
\end{array}\right), \\
h_{a i} & :=\exp \left(a_{a i}+i b_{a i}\right) .
\end{aligned}
$$

The limit of (4.4) as $a_{1 N_{F}-1} \rightarrow-\infty$ is the elementary wall that interpolates $\left\langle N_{F}-4 ; N_{F}-2 ; N_{F}\right\rangle$ and $\left\langle N_{F}-3 ; N_{F}-2 ; N_{F}\right\rangle$. The limit of (4.4) as $a_{1 N_{F}-1} \rightarrow-\infty$

\footnotetext{
${ }^{1}$ This type of three-dimensional diagram is applied to $\mathbf{C} P^{3}$ in [39].
} 


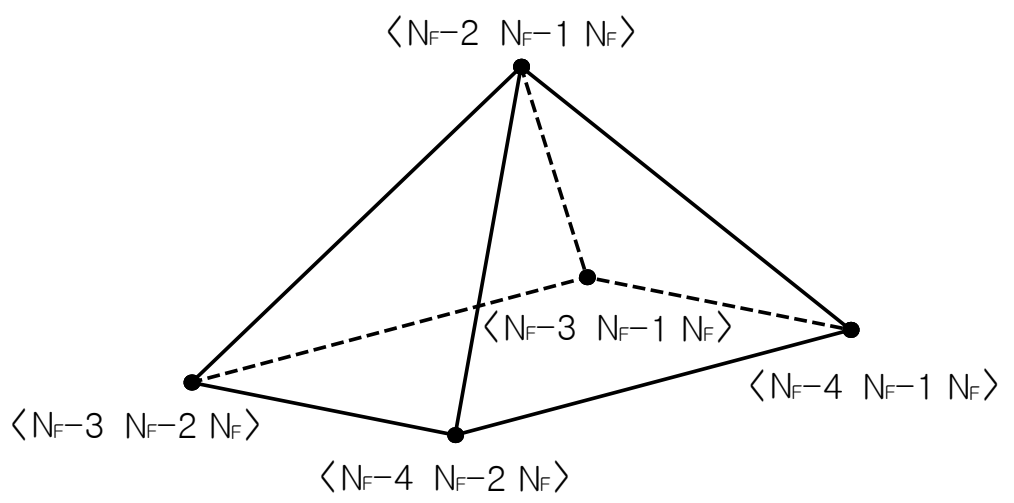

Figure 3. Vertices, edges and triangular faces correspond to vacua, walls and three-pronged junctions. The parallelogram shaped base corresponds to two pairs of penetrable walls.

and $a_{1 N_{F}-3} \rightarrow-\infty$, keeping $a_{1 N_{F}-4}$ finite is $\left\langle N_{F}-4 ; N_{F}-2 ; N_{F}\right\rangle$. The limit of (4.4) as $a_{1 N_{F^{-1}}} \rightarrow-\infty$ and $a_{1 N_{F^{-4}}} \rightarrow-\infty$, keeping $a_{1 N_{F}-3}$ finite is $\left\langle N_{F}-3 ; N_{F}-2 ; N_{F}\right\rangle$. Equation (2.19) with (4.4) is computed as

$$
\begin{aligned}
S S^{\dagger}= & \frac{1}{c} H_{0} e^{\left(2 \widetilde{M}_{1} x^{1}+2 \widetilde{M}_{2} x^{2}\right)} H_{0}^{\dagger}=\frac{1}{c} \operatorname{diag}\left(\Delta_{1}, \Delta_{2}, \Delta_{3}\right), \\
\Delta_{1}= & \exp \left(2 l_{N_{F}-4} x^{1}+2 n_{N_{F}-4} x^{2}+2 a_{1 N_{F}-4}\right)+\exp \left(2 l_{N_{F}-3} x^{1}+2 n_{N_{F}-3} x^{2}+2 a_{1 N_{F}-3}\right) \\
& +\exp \left(2 l_{N_{F}-1} x^{1}+2 n_{N_{F}-1} x^{2}+2 a_{1 N_{F}-1}\right), \\
\Delta_{2}= & \exp \left(2 l_{N_{F}-2} x^{1}+2 n_{N_{F}-2} x^{2}+2 a_{2 N_{F}-2}\right), \\
\Delta_{3}= & \exp \left(2 l_{N_{F}} x^{1}+2 n_{N_{F}} x^{2}+2 a_{3 N_{F}}\right) .
\end{aligned}
$$

The BPS solution (2.17) with (4.4) and (4.5) is

$$
\begin{aligned}
\phi & =S^{-1} H_{0} e^{\widetilde{M}_{1} x^{1}+\widetilde{M}_{2} x^{2}} \\
& =\sqrt{c}\left(\begin{array}{cccccc}
\cdots & \frac{f_{1} N_{F}-4}{\sqrt{\Delta_{1}}} & \frac{f_{1} N_{F}-3}{\sqrt{\Delta_{1}}} & 0 & \frac{f_{1 N_{F}-1}}{\sqrt{\Delta_{1}}} & 0 \\
\cdots & 0 & 0 & \frac{f_{2 N_{F}-2}}{\sqrt{\Delta_{2}}} & 0 & 0 \\
\cdots & 0 & 0 & 0 & 0 & \frac{f_{3 N_{F}}}{\sqrt{\Delta_{3}}}
\end{array}\right), \\
f_{a i} & :=\exp \left(l_{i} x^{1}+n_{i} x^{2}+a_{a i}+i b_{a i}\right) .
\end{aligned}
$$

Component $\left(2, N_{F}-2\right)$ and component $\left(3, N_{F}\right)$ of $(4.6)$ are $\sqrt{c}$ with $\mathrm{U}(1)$ phases. Since the moduli matrix (4.4) has the worldvolume symmetry (2.20), only two of the parameters, $h_{1 i},\left(i=N_{F}-4, N_{F}-3, N_{F}-1\right)$ are independent. It is consistent with the fact that moduli matrix (4.4) consists of three walls and the position of the junction is determined by two of them.

The position of the junction is the solution of the equations:

$$
\operatorname{Re}\left(f_{N_{N_{F}}-4}\right)=\operatorname{Re}\left(f_{1_{N_{F}}-3}\right)=\operatorname{Re}\left(f_{1_{N_{F}}-1}\right),
$$

which produce

$$
\begin{aligned}
& \left(l_{N_{F}-4}-l_{N_{F}-3}\right) x^{1}+\left(n_{N_{F}-4}-n_{N_{F}-3}\right) x^{2}+a_{1 N_{F}-4}-a_{1 N_{F}-3}=0, \\
& \left(l_{N_{F}-3}-l_{N_{F}-1}\right) x^{1}+\left(n_{N_{F}-3}-n_{N_{F}-1}\right) x^{2}+a_{1 N_{F}-3}-a_{1 N_{F}-1}=0 .
\end{aligned}
$$


The position of the junction is

$$
\begin{aligned}
\left(x^{1}, x^{2}\right)= & \left(\frac{S_{1}}{S_{3}}, \frac{S_{2}}{S_{3}}\right) \\
S_{1}= & \left(-n_{N_{F}-3}+n_{N_{F}-1}\right) a_{1_{N_{F}}-4}+\left(-n_{N_{F}-1}+n_{N_{F}-4}\right) a_{1 N_{F}-3} \\
& +\left(-n_{N_{F}-4}+n_{N_{F}-3}\right) a_{1 N_{F}-1}, \\
S_{2}= & \left(l_{N_{F}-3}-l_{N_{F}-1}\right) a_{1 N_{F}-4}+\left(-l_{N_{F}-4}+l_{N_{F}-1}\right) a_{1 N_{F}-3} \\
& +\left(l_{N_{F}-4}-l_{N_{F}-3}\right) a_{1 N_{F}-1}, \\
S_{3}= & \left(-l_{N_{F}-3}+l_{N_{F}-1}\right) n_{N_{F}-4}+\left(-l_{N_{F}-1}+l_{N_{F}-4}\right) n_{N_{F}-3} \\
& +\left(-l_{N_{F}-4}+l_{N_{F}-3}\right) n_{N_{F}-1} .
\end{aligned}
$$

As mentioned previously, only two of the parameters, $a_{1 i},\left(i=N_{F}-4, N_{F}-3, N_{F}-1\right)$ are independent.

The matrix $S S^{\dagger}$ defined by (2.19) is not diagonal for non-Abelian three-pronged junctions as the vacua differ by two label components. A detour is needed to apply the moduli matrix formalism to junctions. In [40], the Grassmann manifold is embedded into the complex projective space by the Plücker embedding. In [42], the moduli matrix formalism is applied to each wall that form a three-pronged junction as the set of walls can be identified in the diagrams of the pictorial representation.

We study non-Abelian three-pronged junction $\left\{\left\langle N_{F}-4 ; N_{F}-2 ; N_{F}\right\rangle,\left\langle N_{F}-4 ; N_{F}-\right.\right.$ $\left.\left.1 ; N_{F}\right\rangle,\left\langle N_{F}-2 ; N_{F}-1 ; N_{F}\right\rangle\right\}$ following the method of [42]. The moduli matrix of the wall, which interpolates $\left\langle N_{F}-4 ; N_{F}-2 ; N_{F}\right\rangle$ and $\left\langle N_{F}-4 ; N_{F}-1 ; N_{F}\right\rangle$ is

$$
\begin{aligned}
H_{0} & =\left(\begin{array}{cccccc}
\cdots & h_{1 N_{F}-4} & 0 & 0 & 0 & 0 \\
\cdots & 0 & 0 & h_{2 N_{F}-2} & h_{2 N_{F}-1} & 0 \\
\cdots & 0 & 0 & 0 & 0 & h_{3 N_{F}}
\end{array}\right), \\
h_{a i} & :=\exp \left(a_{a i}+i b_{a i}\right) .
\end{aligned}
$$

The wall solution is

$$
\begin{aligned}
& \phi=S^{-1} H_{0} e^{\widetilde{M}_{1} x^{1}+\widetilde{M}_{2} x^{2}} \\
& =\sqrt{c}\left(\begin{array}{cccccc}
\cdots & \frac{f_{1} N_{F}-4}{\sqrt{\Delta_{1}}} & 0 & 0 & 0 & 0 \\
\cdots & 0 & 0 & \frac{f_{2 N_{F}-2}}{\sqrt{\Delta_{2}}} & \frac{f_{2 N_{F}-1}}{\sqrt{\Delta_{2}}} & 0 \\
\cdots & 0 & 0 & 0 & 0 & \frac{f_{3 N_{F}}}{\sqrt{\Delta_{3}}}
\end{array}\right), \\
& f_{a i}:=\exp \left(l_{i} x^{1}+n_{i} x^{2}+a_{a i}+i b_{a i}\right), \\
& \Delta_{1}=\exp \left(2 l_{N_{F}-4} x^{1}+2 n_{N_{F}-4} x^{2}+2 a_{1 N_{F}-4}\right) \text {, } \\
& \Delta_{2}=\exp \left(2 l_{N_{F}-2} x^{1}+2 n_{N_{F}-2} x^{2}+2 a_{2 N_{F}-2}\right) \\
& +\exp \left(2 l_{N_{F}-1} x^{1}+2 n_{N_{F}-1} x^{2}+2 a_{2 N_{F}-1}\right), \\
& \Delta_{3}=\exp \left(2 l_{N_{F}} x^{1}+2 n_{N_{F}} x^{2}+2 a_{3 N_{F}}\right) \text {. }
\end{aligned}
$$

Component $\left(1, N_{F}-4\right)$ and component $\left(3, N_{F}\right)$ of $(4.12)$ are $\sqrt{c}$ with $\mathrm{U}(1)$ phases. Since moduli matrix (4.11) has the worldvolume symmetry (2.20), only one of the parameters, $h_{2 i}$, 
( $\left.i=N_{F}-2, N_{F}-1\right)$ is independent. The wall is located in the region where $\operatorname{Re}\left(f_{2 N_{F}-2}\right)=$ $\operatorname{Re}\left(f_{2 N_{F}-1}\right)$ :

$$
\left(l_{N_{F}-2}-l_{N_{F}-1}\right) x^{1}+\left(n_{N_{F}-2}-n_{N_{F}-1}\right) x^{2}+\left(a_{2 N_{F}-2}-a_{2 N_{F}-1}\right)=0 .
$$

Only one of the parameters, $a_{2 i},\left(i=N_{F}-2, N_{F}-1\right)$ is independent.

The moduli matrix of the wall that interpolates $\left\langle N_{F}-4 ; N_{F}-1 ; N_{F}\right\rangle$ and $\left\langle N_{F}-\right.$ $\left.2 ; N_{F}-1 ; N_{F}\right\rangle$ is

$$
H_{0}=\left(\begin{array}{cccccc}
\cdots & h_{1 N_{F}-4} & 0 & h_{1 N_{F}}-2 & 0 & 0 \\
\cdots & 0 & 0 & 0 & h_{2 N_{F}-1} & 0 \\
\cdots & 0 & 0 & 0 & 0 & h_{3 N_{F}}
\end{array}\right), \quad h_{a i}:=e^{\left(a_{a i}+i b_{a i}\right)}
$$

and the position of the wall is

$$
\left(l_{N_{F}-4}-l_{N_{F}-2}\right) x^{1}+\left(n_{N_{F}-4}-n_{N_{F}-2}\right) x^{2}+a_{1 N_{F}-4}-a_{1 N_{F}-2}=0 .
$$

The moduli matrix of the wall that interpolates $\left\langle N_{F}-2 ; N_{F}-1 ; N_{F}\right\rangle$ and $\left\langle N_{F}-4 ; N_{F}-\right.$ $\left.2 ; N_{F}\right\rangle$ is

$$
H_{0}=\left(\begin{array}{cccccc}
\cdots & 0 & 0 & h_{1 N_{F}-2} & 0 & 0 \\
\cdots & h_{2 N_{F}-4} & 0 & 0 & h_{2 N_{F}-1} & 0 \\
\cdots & 0 & 0 & 0 & 0 & h_{3 N_{F}}
\end{array}\right), \quad h_{a i}:=e^{\left(a_{a i}+i b_{a i}\right)}
$$

and the position of the wall is

$$
\left(l_{N_{F}-4}-l_{N_{F}-1}\right) x^{1}+\left(n_{N_{F}-4}-n_{N_{F}-1}\right) x^{2}+\left(a_{2 N_{F}-4}-a_{2 N_{F}-1}\right)=0 .
$$

The position of the junction can be calculated by solving the equations (4.13), (4.15) and (4.17). The consistency condition is

$$
a_{1 N_{F}-4}-a_{1 N_{F}-2}=a_{2 N_{F}-4}-a_{2 N_{F}-2} .
$$

Therefore there are two independent parameters for the three-pronged junction as expected. The position of the junction is

$$
\begin{aligned}
\left(x^{1}, x^{2}\right)= & \left(\frac{T_{1}}{T_{3}}, \frac{T_{2}}{T_{3}}\right) \\
T_{1}= & \left(n_{N_{F}-2}-n_{N_{F}-1}\right) a_{2 N_{F}-4}+\left(n_{N_{F}-1}-n_{N_{F}-4}\right) a_{2 N_{F}-2} \\
& +\left(n_{N_{F}-4}-n_{N_{F}-2}\right) a_{2_{N_{F}}-1}, \\
T_{2}= & \left(-l_{N_{F}-2}+l_{N_{F}-1}\right) a_{2_{N_{F}}-4}+\left(-l_{N_{F}-1}+l_{N_{F}-4}\right) a_{2 N_{F}-2} \\
& +\left(-l_{N_{F}-4}+l_{N_{F}-2}\right) a_{2 N_{F}-1}, \\
T_{3}= & \left(l_{N_{F}-2}-l_{N_{F}-1}\right) n_{N_{F}-4}+\left(l_{N_{F}-1}-l_{N_{F}-4}\right) n_{N_{F}-2} \\
& +\left(l_{N_{F}-4}-l_{N_{F}-2}\right) n_{N_{F}-1} .
\end{aligned}
$$

We have computed junction positions of an Abelian three-pronged junction and a nonAbelian three-pronged junction in the pictorial representation by using $N_{C} \times N_{F}$ moduli matrices instead of using the Plücker embedding. 


\section{Summary and discussion}

We have discussed vacua and BPS objects of the $\mathcal{N}=2$ mass-deformed nonlinear sigma models on the Grassmann manifold $G_{N_{F}, N_{C}}$ with $N_{C}=1,2,3$ as an extension of [42]. We have applied the moduli matrix formalism $[19,20]$ and the pictorial representation $[23$, 24, 42] to vacua, walls and three-pronged junctions. Since we can analyse three-pronged junctions with $N_{C} \times N_{F}$ moduli matrices in the pictorial representation, we can apply the moduli matrix formalism to three-pronged junctions of the nonlinear sigma models on $\mathrm{SO}(2 N) / \mathrm{U}(N)$ and $\mathrm{Sp}(N) / \mathrm{U}(N)$, which are quadrics of the Grassmann manifold. We hope to report on the results elsewhere.

We have considered the mass-deformed hyper-Kähler nonlinear sigma models with eight supercharges on the Grassmann manifold in four dimensions. The target space of hypermultiplets for locally supersymmetric nonlinear sigma models with eight supercharges in the dimension $\mathrm{d} \leq 6$ are quaternionic Kähler manifolds [47]. Exact BPS domain wall solutions are studied in five dimensional supergravity coupled with the hypermultiplets and vector multiplet [48]. It is worth considering whether we can improve the method proposed in this work to apply it to the supergravity models.

\section{Acknowledgments}

The author would like to thank A. Golubtsova for early participation and D. Bykov for helpful comments. The author also would like to thank Bogoliubov Laboratory of Theoretical Physics, Joint Institute for Nuclear Research for warm hospitality. This research was supported by Basic Science Research Program through the National Research Foundation of Korea (NRF-2017R1D1A1B03034222).

\section{A Diagram for vacua and elementary walls of the mass-deformed non- linear sigma models on $G_{8,4}$}

We present the diagram for vacua and elementary walls of the mass-deformed nonlinear sigma models on the Grassmann manifold $G_{8,4}$ in figure 4. The manifold $G_{8,4}$ is self-dual under $N_{F} \leftrightarrow N_{F}-N_{C}$. Therefore the diagram has a $\pi$-rotation symmetry.

In figure 1 (c), we have presented the diagram for $G_{7,3}$. The diagram for $G_{7,4}$ is obtained by a $\pi$-rotation of the diagram in figure $1(\mathrm{c})$. Non-orthogonal elementary walls are aligned next to each other while orthogonal elementary walls form parallelograms. By adding $\alpha_{7}$ vectors to proper positions of the diagram for $G_{7,4}$, we can build the diagram for $G_{8,4}$, which is presented in figure 4.

Open Access. This article is distributed under the terms of the Creative Commons Attribution License (CC-BY 4.0), which permits any use, distribution and reproduction in any medium, provided the original author(s) and source are credited. 


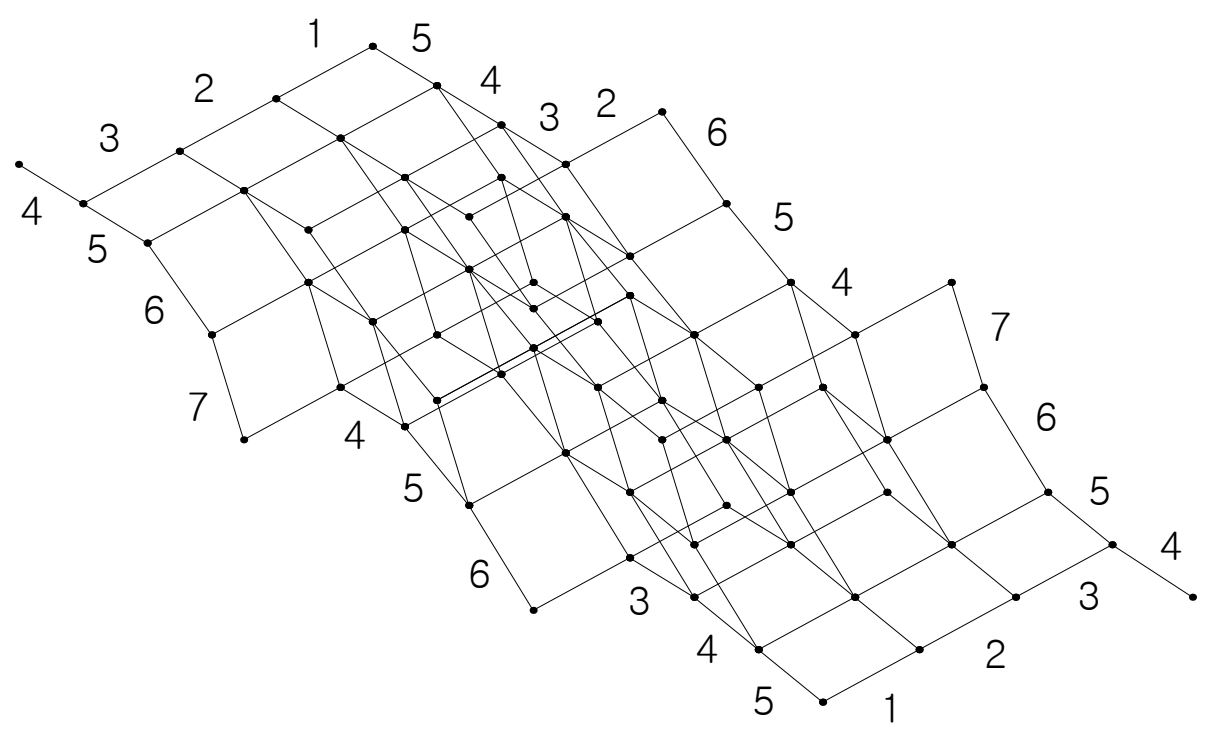

Figure 4. Vacua and elementary walls in $G_{8,4}$. The numbers indicate the subscript $i$ 's of simple $\operatorname{roots} \underline{\alpha}_{i}$.

\section{References}

[1] N. Arkani-Hamed, S. Dimopoulos and G.R. Dvali, The hierarchy problem and new dimensions at a millimeter, Phys. Lett. B 429 (1998) 263 [hep-ph/9803315] [InSPIRE].

[2] I. Antoniadis, N. Arkani-Hamed, S. Dimopoulos and G.R. Dvali, New dimensions at a millimeter to a Fermi and superstrings at a TeV, Phys. Lett. B 436 (1998) 257 [hep-ph/9804398] [INSPIRE].

[3] L. Randall and R. Sundrum, A large mass hierarchy from a small extra dimension, Phys. Rev. Lett. 83 (1999) 3370 [hep-ph/9905221] [INSPIRE].

[4] L. Randall and R. Sundrum, An alternative to compactification, Phys. Rev. Lett. 83 (1999) 4690 [hep-th/9906064] [INSPIRE].

[5] M. Bucher and D.N. Spergel, Is the dark matter a solid?, Phys. Rev. D 60 (1999) 043505 [astro-ph/9812022] [INSPIRE].

[6] R.A. Battye, M. Bucher and D. Spergel, Domain wall dominated universes, astro-ph/9908047 [INSPIRE].

[7] L. Conversi, A. Melchiorri, L. Mersini-Houghton and J. Silk, Are domain walls ruled out?, Astropart. Phys. 21 (2004) 443 [astro-ph/0402529] [INSPIRE].

[8] A. Friedland, H. Murayama and M. Perelstein, Domain walls as dark energy, Phys. Rev. D 67 (2003) 043519 [astro-ph/0205520] [INSPIRE].

[9] J.C. R.E. Oliveira, C.J. A.P. Martins and P.P. Avelino, The cosmological evolution of domain wall networks, Phys. Rev. D 71 (2005) 083509 [hep-ph/0410356] [INSPIRE].

[10] B.M. Roberts et al., Search for domain wall dark matter with atomic clocks on board global positioning system satellites, Nature Commun. 8 (2017) 1195 [arXiv:1704.06844] [INSPIRE].

[11] E.R.C. Abraham and P.K. Townsend, Q kinks, Phys. Lett. B 291 (1992) 85 [INSPIRE]. 
[12] E.R.C. Abraham and P.K. Townsend, More on Q kinks: a $(1+1)$-dimensional analog of dyons, Phys. Lett. B 295 (1992) 225 [INSPIRE].

[13] J.P. Gauntlett, D. Tong and P.K. Townsend, Multidomain walls in massive supersymmetric $\sigma$-models, Phys. Rev. D 64 (2001) 025010 [hep-th/0012178] [INSPIRE].

[14] D. Tong, The moduli space of BPS domain walls, Phys. Rev. D 66 (2002) 025013 [hep-th/0202012] [INSPIRE].

[15] M. Arai, M. Naganuma, M. Nitta and N. Sakai, Manifest supersymmetry for BPS walls in $N=2$ nonlinear $\sigma$-models, Nucl. Phys. B 652 (2003) 35 [hep-th/0211103] [InSPIRE].

[16] M. Arai, E. Ivanov and J. Niederle, Massive nonlinear $\sigma$-models and BPS domain walls in harmonic superspace, Nucl. Phys. B 680 (2004) 23 [hep-th/0312037] [INSPIRE].

[17] Y. Isozumi, K. Ohashi and N. Sakai, Massless localized vector field on a wall in D $=5$ SQED with tensor multiplets, JHEP 11 (2003) 061 [hep-th/0310130] [INSPIRE].

[18] Y. Isozumi, K. Ohashi and N. Sakai, Exact wall solutions in five-dimensional SUSY QED at finite coupling, JHEP 11 (2003) 060 [hep-th/0310189] [INSPIRE].

[19] Y. Isozumi, M. Nitta, K. Ohashi and N. Sakai, Construction of non-Abelian walls and their complete moduli space, Phys. Rev. Lett. 93 (2004) 161601 [hep-th/0404198] [INSPIRE].

[20] Y. Isozumi, M. Nitta, K. Ohashi and N. Sakai, Non-Abelian walls in supersymmetric gauge theories, Phys. Rev. D 70 (2004) 125014 [hep-th/0405194] [INSPIRE].

[21] K. Higashijima and M. Nitta, Supersymmetric nonlinear $\sigma$-models as gauge theories, Prog. Theor. Phys. 103 (2000) 635 [hep-th/9911139] [INSPIRE].

[22] N. Sakai and D. Tong, Monopoles, vortices, domain walls and D-branes: the rules of interaction, JHEP 03 (2005) 019 [hep-th/0501207] [INSPIRE].

[23] B.-H. Lee, C. Park and S. Shin, Vacua and walls of mass-deformed Kähler nonlinear $\sigma$-models on $\mathrm{SO}(2 N) / \mathrm{U}(N)$, Phys. Rev. D 96 (2017) 105017 [arXiv: 1708.05243] [INSPIRE].

[24] M. Arai, A. Golubtsova, C. Park and S. Shin, Vacua and walls of mass-deformed Kähler nonlinear $\sigma$-models on $\mathrm{Sp}(N) / \mathrm{U}(N)$, Phys. Rev. D 97 (2018) 105012 [arXiv:1803.09275] [INSPIRE].

[25] A. Galperin, E. Ivanov, V. Ogievetsky and P.K. Townsend, Eguchi-Hanson type metrics from harmonic superspace, Class. Quant. Grav. 3 (1986) 625

[26] A. Galperin, E. Ivanov, V. Ogievetsky and E. Sokatchev, HyperKähler metrics and harmonic superspace, Commun. Math. Phys. 103 (1986) 515 [INSPIRE].

[27] A.S. Galperin, E.A. Ivanov, V.I. Ogievetsky and E.S. Sokatchev, Harmonic superspace, Cambridge University Press, Cambridge U.K. (2001).

[28] M. Arai, S.M. Kuzenko and U. Lindström, Hyper-Kähler $\sigma$-models on cotangent bundles of Hermitian symmetric spaces using projective superspace, JHEP 02 (2007) 100 [hep-th/0612174] [INSPIRE].

[29] E.R.C. Abraham and P.K. Townsend, Intersecting extended objects in supersymmetric field theories, Nucl. Phys. B 351 (1991) 313 [INSPIRE].

[30] G.W. Gibbons and P.K. Townsend, A Bogomolny equation for intersecting domain walls, Phys. Rev. Lett. 83 (1999) 1727 [hep-th/9905196] [INSPIRE]. 
[31] S.M. Carroll, S. Hellerman and M. Trodden, Domain wall junctions are 1/4-BPS states, Phys. Rev. D 61 (2000) 065001 [hep-th/9905217] [InSPIRE].

[32] P.M. Saffin, Tiling with almost BPS junctions, Phys. Rev. Lett. 83 (1999) 4249 [hep-th/9907066] [INSPIRE].

[33] A. Gorsky and M.A. Shifman, More on the tensorial central charges in $N=1$ supersymmetric gauge theories (BPS wall junctions and strings), Phys. Rev. D 61 (2000) 085001 [hep-th/9909015] [INSPIRE].

[34] H. Oda, K. Ito, M. Naganuma and N. Sakai, An exact solution of BPS domain wall junction, Phys. Lett. B 471 (1999) 140 [hep-th/9910095] [INSPIRE].

[35] J.P. Gauntlett, D. Tong and P.K. Townsend, Supersymmetric intersecting domain walls in massive hyper-Kähler $\sigma$-models, Phys. Rev. D 63 (2001) 085001 [hep-th/0007124] [INSPIRE].

[36] S. Nam and K. Olsen, Domain wall junctions in supersymmetric field theories in $D=4$, JHEP 08 (2000) 001 [hep-th/0002176] [INSPIRE].

[37] D. Bazeia and F.A. Brito, Bags, junctions and networks of BPS and nonBPS defects, Phys. Rev. D 61 (2000) 105019 [hep-th/9912015] [INSPIRE].

[38] D. Binosi and T. ter Veldhuis, Domain wall junctions in a generalized Wess-Zumino model, Phys. Lett. B 476 (2000) 124 [hep-th/9912081] [INSPIRE].

[39] M. Eto et al., Webs of walls, Phys. Rev. D 72 (2005) 085004 [hep-th/0506135] [InSPIRE].

[40] M. Eto et al., Non-Abelian webs of walls, Phys. Lett. B 632 (2006) 384 [hep-th/0508241] [INSPIRE].

[41] M. Eto et al., Dynamics of domain wall networks, Phys. Rev. D 76 (2007) 125025 [arXiv: 0707.3267] [INSPIRE].

[42] S. Shin, Vacua, walls and junctions in $G_{N_{F}, N_{C}}$, Nucl. Phys. B 946 (2019) 114701 [arXiv: 1804.05822] [INSPIRE].

[43] M. Rocek and P.K. Townsend, Three loop finiteness of the $N=4$ supersymmetric nonlinear sigma model, Phys. Lett. B 96 (1980) 72.

[44] U. Lindström and M. Roček, Scalar tensor duality and $N=1, N=2$ nonlinear $\sigma$-models, Nucl. Phys. B 222 (1983) 285 [inSPIRE].

[45] M. Arai, M. Nitta and N. Sakai, Vacua of massive hyper-Kähler $\sigma$-models of non-Abelian quotient, Prog. Theor. Phys. 113 (2005) 657 [hep-th/0307274] [INSPIRE].

[46] A. Isaev and V. Rubakov, Theory of groups and symmetries, World Scientific, Singapore (2018).

[47] J. Bagger and E. Witten, Matter couplings in $N=2$ supergravity, Nucl. Phys. B 222 (1983) 1 [INSPIRE].

[48] M. Arai, S. Fujita, M. Naganuma and N. Sakai, Wall solution with weak gravity limit in five-dimensional supergravity, Phys. Lett. B 556 (2003) 192 [hep-th/0212175] [INSPIRE]. 